Workshop on Econophysics of the

International Conference on Statistical Physics (SigmaPhi2011)

International Journal of Modern Physics: Conference Series

Vol. 16 (2012) 41-50

(C) World Scientific Publishing Company

DOI: $10.1142 /$ S2010194512007763

\title{
EFFECTS OF INTRADAY PATTERNS ON ANALYSIS OF STOCK MARKET INDEX AND TRADING VOLUME
}

\author{
HYUNG WOOC CHOI, SEONG EUN MAENG and JAE WOO LEE* \\ Department of Physics, Inha University, 100 Inharo \\ Incheon, 402-751, Korea \\ *jaewlee@inha.ac.kr
}

\begin{abstract}
We review the stylized properties of the stock market and consider effects of the intraday patterns on the analysis of the time series for the stock index and the trading volume in Korean stock market. In the stock market the probability distribution function (pdf) of the return and volatility followed the power law for the stock index and the change of the volume traded. The volatility of the stock index showed the long-time memory and the autocorrelation function followed a power law. We applied two eliminating methods of the intraday patterns: the intraday patterns of the time series itself, and the intraday patterns of the absolute return for the index or the absolute volume change. We scaled the index and return by two types of the intraday patterns. We considered the probability distribution function and the autocorrelation function (ACF) for the time series scaled by the intraday patterns. The cumulative probability distribution function of the returns scaled by the intraday patterns showed a power law, $P_{>}(r) \sim r^{-\alpha_{ \pm}}$, where $\alpha_{ \pm}$ corresponds to the exponent of the positive and negative fat tails. The pdf of the return scaled by intraday patterns by the absolute return decayed much steeper than that of the return scaled by intraday patterns of the index itself. The pdf for the volume change also followed the power law for both methods of eliminating intraday patterns. However, the exponents of the power law at fat tails do not depend on the intraday patterns. The $\mathrm{ACF}$ of the absolute return showed long-time correlation and followed the power law for the scaled index and for the scaled volume. The daily periodicity of the ACF was removed for scaled time series by the intraday patterns of the absolute return or the absolute volume change.
\end{abstract}

Keywords: Stock market; intraday patterns; trading volume; power law.

\section{Introduction}

The statistical physical and dynamic properties of the stock market have been extensively studied last two decades ${ }^{1,2,3,4,5,6}$. Many stylized facts reported for the stock market index and trading volume, foreign exchange rate, etc. $7,8,9,10,11,12,13,14,15,16,17,18,19,20,21$. The short range correlations observed for the autocorrelation of the return, The autocorrelation of the absolute return or volatility showed the long-range correlations and followed a power law. The fluctuations in the financial time series exist in the volatility. This long time memory influences the time behaviors of the stock market. Therefore, the prediction of the stock index is 
impossible when we don't know the full informations of the volatility and upcoming news which influence strongly to the market. The probability distribution function of the return and the volatility deviate from the normal probability distribution function and show the power law at the fat tail. The fat tail of the distribution function is a proof of the self-organized criticality in the stock market. The stock market evolves by the internal interactions among the agents in the stock market and the external impacts.

When we analyzed the time series of the stock index or the trading volume, we eliminated the intraday patterns of activity. In the stock market the heaviest trading activities exist around opening and closing hours of the market. Some studies have removed intraday or weekly patterns from stock-index and stock-volume analysis ${ }^{21,22,23,24}$. Wood et al. reported that all positive returns were earned during the first thirty minutes and at the market close. When the first thirty minutes and closing minutes were excluded, market returns followed the Gaussian function, and autocorrelation was found to be substantially reduced ${ }^{26}$. Wang et al. studied the scaling properties of the intraday volatility return interval in US stock markets ${ }^{23}$. Liu et al. removed the intraday pattern in New York Stock Exchange (NYSE) and Standard and Poor (S \& P) 500 index when they analyzed correlation functions of the return and volatility ${ }^{21}$. Many analyses do not eliminate intraday patterns when they obtain the stylized properties. In the present work's time series analysis, we introduce two types of eliminating methods of the daily pattern for the index and the trading volume. As there is no one absolute method of stock-market time-series analysis, two types of intraday pattern were considered: those of the index and trading volume itself, and those of the absolute return (or volatility) and absolute volume change. In section 2 we review the stylized properties of the time series in the stock market. In section 3 we present the effects of the intraday patterns in the analysis of the time series. The conclusion is given in section 4 .

\section{Stylized Properties of Stock Market}

The economic time series show the intrinsic stylized properties owing to its own dynamics. The stock markets are influenced by many economic agents such as individual, companies, brokers, fund managers, foreign exchange rate etc. The dynamics of the stock market is basically non-linear and multiscaling.

The stock market index and the trading volume are represented by $I(t)$ and $Q(t)$ at time $t$, respectively. The simple returns of the stock market is defined as

$$
x(t)=I(t+\Delta t)-I(t),
$$

where $\Delta t$ is the time lag. The logarithmic return is defined as

$$
r(t)=\ln I_{j}(t+\Delta t)-\ln I_{j}(t),
$$

where $j$ indicates a particular trading day. Similarly, we define the trading volume change as

$$
q(t)=\ln Q_{j}(t+\Delta t)-\ln Q_{j}(t)
$$


The probability distribution function (pdf) deviates from the normal distribution function with fat tails. The skewness, $k_{s}$, and the kurtosis, $\kappa$, of the distribution function are defined as,

$$
\begin{gathered}
k_{s}=\frac{<(r(t)-<r>)^{3}>}{\sigma^{3}}, \\
\kappa=\frac{<(r(t)-<r>)^{4}>}{\sigma^{4}}-3,
\end{gathered}
$$

where $\sigma^{2}(\Delta t)$ is the variance of the logarithmic return. For the normal distribution function, the kurtosis is zero $(\kappa=0)$. A positive value of $\kappa$ indicates the fat tail of the distribution function. For many financial time series, the positive kurtosis was observed $^{17}$. In the Korean stock market index with $\Delta t=1 \mathrm{~min}$, we observed the positive kurtosis $\kappa=780^{7,8}$. The pdf of the return in the stock market showed the negative value of the skewness ${ }^{8}$. The negative skewness is called gain-loss asymme$\operatorname{try}^{8}$.

The pdf of the return followed a non-Gaussian distribution and showed fat tails in the stock markets ${ }^{21}$. The probability distribution function of the return for the positive and negative tail follows a power law,

$$
P(r) \sim r^{-\alpha^{ \pm}(\Delta t)-1}
$$

where the exponents, $\alpha^{ \pm}(\Delta t)$, depend on the time lag $\Delta t$ when we define the return For many stock markets, the exponents, $\alpha^{ \pm}(\Delta t)$, were greater than two ${ }^{17,21}$. The cumulative pdf of the volume traded obeys a power law ${ }^{12}$,

$$
P_{>}(Q>y) \sim y^{-\alpha_{Q}} .
$$

The exponent, $\alpha_{Q}$, is non-universal behavior and depends on the stock market: $\alpha_{Q}=1.7$ in the US stock market ${ }^{9,27}, \alpha_{Q}=1$ in the Japanese stock market ${ }^{28}$, $\alpha_{Q}=2.69$ in the Korean stock market ${ }^{12}$. The cumulative probability distribution function of the absolute logarithmic price change, $|r(t)|$, follows a power law,

$$
P_{>}(|r|>y) \sim y^{-\beta(\Delta t)},
$$

where the exponent, $\beta(\Delta t)$, characterize the decaying speed of the fat tail. It was suggested that the cumulative pdf of the absolute return follows the inverse-cubic law, $\beta(\Delta t)=3^{19}$. The cumulative probability distribution function of the absolute volume change follows a power law,

$$
P_{>}(|q|>y) \sim y^{-\beta_{q}(\Delta t)} .
$$

The exponent, $\beta_{q}$, does not show the universal behavior. It depends on the stock market and the time lag $\Delta t$. In the US stock market the exponent was observed as $\beta_{q}=3$ for $\mathrm{S} \& \mathrm{P} 500$ index, and $\beta_{q}=4.2$ for the NYSE composite index ${ }^{19}$. Hong et al. showed that the exponent, $\beta_{q}$, depends on the time lag in the Korean stock market $^{12}$. 
Another stylized property is the correlation relation in the stock market. The return has a short memory and the autocorrelation, $C_{r}(T)$, decays exponentially.

$$
C_{r}(T)=<r(t+T) r(t)>\sim \exp (-T / \tau),
$$

where the characteristic time $\tau$ is the order of $10 \mathrm{~min}^{21}$. The long memory in the stock index exists in the absolute return or in the volatility. We define the autocorrelation function of the absolute return as

$$
C_{|r|}(t)=<\left|r\left(t^{\prime}+t\right)\right|\left|r\left(t^{\prime}\right)\right|>-<\left|r\left(t^{\prime}\right)\right|^{2}>.
$$

The autocorrelation of the volatility followed a power law,

$$
C_{|r|}(t) \sim t^{-\gamma}
$$

in the stock market ${ }^{8}$. The exponent $\gamma$ was $\gamma=0.3$ in S \& P $500^{19}$.

In the stock market many agents interact each other. Therefore, we can not expect a unique scaling measure. We define the generalized $q$ th-order autocorrelation functions in the absolute return as

$$
F_{q}(\Delta t)=<\left|r_{\Delta t}(t)\right|^{q}>
$$

The generalized $q$ th order autocorrelation function showed the power law,

$$
F_{q} \sim \Delta t^{\tau(q)},
$$

where the scaling exponents $\tau(q)$ are a non-linear function of $q$. If the exponent $\tau(q)$ is independent on $q$, then the time series is monofractal. If the exponent $\tau(q)$ is the non-linear function of $q$, the time series have multiscaling and are called multifractal. The time series of the stock index showed the multifractality ${ }^{8}$.

\section{Intraday Effects on Analysis of Stock Index}

This study is of one-minute data from the Korean composite stock price index (KOSPI) from January 2, 2004, to October 30, 2009. Only trading hours were considered: closing hours, weekends and holidays were removed from the data. The total number of data was $\mathrm{N}=508,984$.

We consider two types of the intraday patterns. One is the intraday patterns of the index itself and the intraday pattern of the volume. The intraday patterns of the index itself is defined by

$$
I_{d a y}(t)=\sum_{j=1}^{N_{d a y}} \frac{I_{j}(t)}{N_{d a y}} .
$$

Similarly, the intraday patterns of the volume itself is defined by

$$
Q_{d a y}(t)=\sum_{j=1}^{N_{d a y}} \frac{Q_{j}(t)}{N_{d a y}} .
$$

The other is the intraday patterns of the volatility for the index and the intraday patterns of the absolute volume change. We defined the intraday patterns of the 
volatility for the index as

$$
|r|_{\text {day }}(t)=\sum_{j=1}^{N_{d a y}} \frac{\left|r_{j}(t)\right|}{N_{d a y}} .
$$

The intraday patterns of the absolute volume change is defined by

$$
|q|_{\text {day }}(t)=\sum_{j=1}^{N_{d a y}} \frac{\left|q_{j}(t)\right|}{N_{d a y}} .
$$

We show the intraday patterns of the index itself at Fig. 1 (a) and the intraday patterns of the volume itself at Fig. 1 (b). We observed that the heavy trades occur around opening and closing hours of the market. We also show the daily patterns of the volatility for the index at Fig. 1 (c) and the daily patterns of the absolute volume change at Fig. 1 (d). The large fluctuations and the large changes of trading volume were observed around the opening and closing hours. The volatilities were stabilized after 30 minutes of the opening hour. We eliminated the daily patterns from the index and the trading volume by using two methods. We consider three types of the return: $r_{\text {org }}(t)$ represents the return of the original index, $r_{\text {org }}(t)=r(t)$,
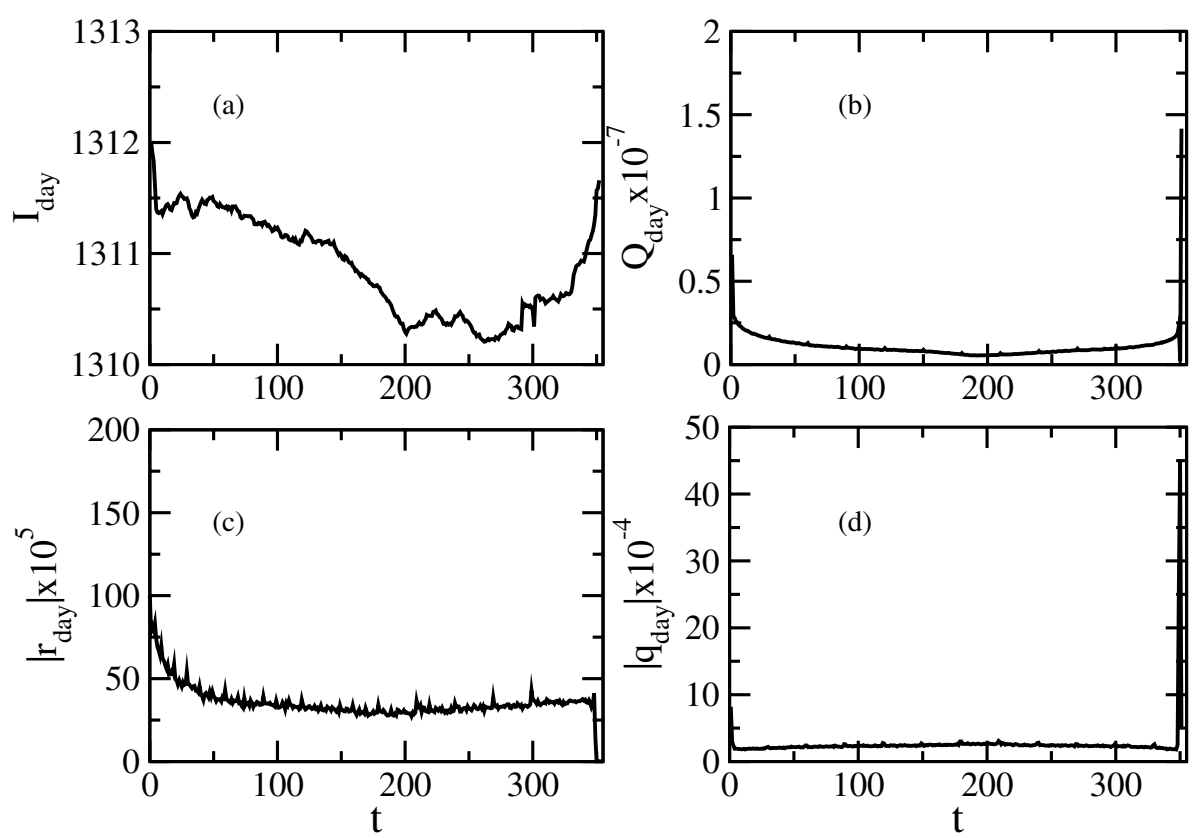

Fig. 1. Intraday patterns of the stock index and the trading volume. (a) Daily patterns of the index itself, (b) daily patterns of the trading volume, (c) daily patterns of the volatility, and (d) daily patterns of the absolute volume change. In the Korean stock market, the market opens on 9:00 and closes on 15:00. The large trades occur around opening and closing hours. 
the return of the scaled index by the intraday pattern of the index itself,

$$
r_{d a y}(t)=\ln \left(\frac{I_{j}(t+1)}{I_{d a y}}\right)-\ln \left(\frac{I_{j}(t)}{I_{d a y}}\right),
$$

and the return of the scaled by the intraday pattern of the volatility,

$$
r_{v o l}(t)=\frac{r(t)}{|r|_{\text {day }}} .
$$

In Fig. 2 we presented the probability density function for three types of the return. The pdfs of the returns for $r_{\text {org }}$ and $r_{d a y}$ are different from those for $r_{v o l}$. The pdfs for the return $r_{\text {org }}$ and $r_{d a y}$ coincide, but the pdf of the return $r_{v o l}$ is different to the previous types of the return. The tail parts of the pdfs show the fat tails. Inset in Fig. 2 represents the $\log$-log plot of the positive fat tails for the cumulative pdf. The tails show the power law such as $P_{>}(r) \sim r^{-\alpha}$. The power law exponent of the pdf for $r_{v o l}$ is larger than those for $r_{o r g}$ and $r_{d a y}$. We obtained the exponent of the power law, $\alpha($ org $)=\alpha($ day $)=2.66(3)$, and $\alpha(v o l)=4.24(6)$

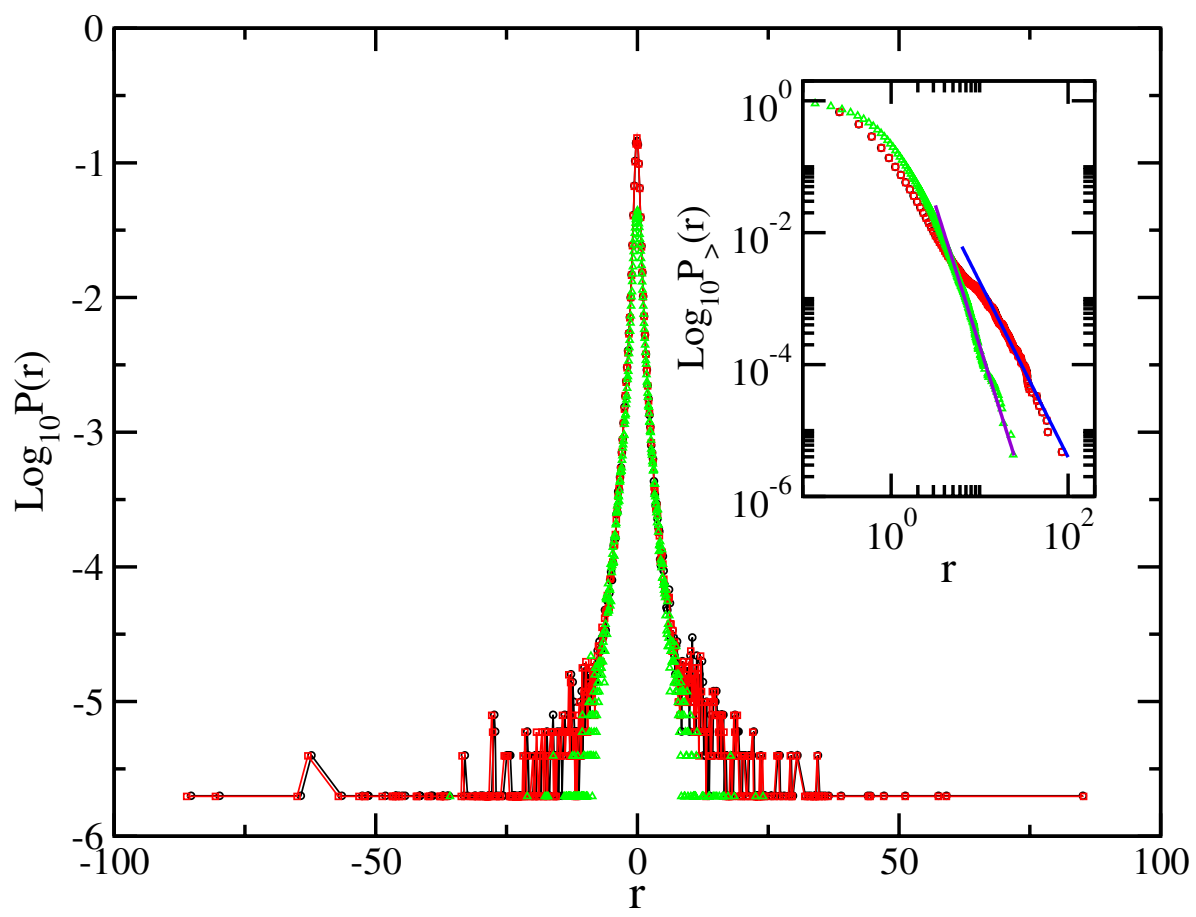

Fig. 2. Log-linear plot of the probability density function for the return of the original index (circle), $r_{\text {org }}$, the return of the scaled index by the intraday pattern of the index itself (square), $r_{d a y}$, and the scaled return by the volatility (triangle), $r_{v o l}$. The pdfs for the return $r_{o r g}$ and $r_{d a y}$ coincide, but the pdf of the return $r_{v o l}$ is different. The tail parts of the pdfs show the fat tails. Inset represents the log-log plot of the positive fat tails for the pdf. The tails show the power law. The power exponent of the pdf for $r_{v o l}$ is larger than those for $r_{o r g}$ and $r_{d a y}$. 
for the positive tails, and $\alpha($ org $)=\alpha($ day $)=2.15(4)$, and $\alpha(v o l)=4.20(7)$ for the negative tails

In Fig. 3 we presented the probability density function for the volume change. For the volume change of the original trading volume, we observed the second peak around $q=10$. This second peak disappeared for the volume change scaled by the intraday pattern of the trading volume itself (square), $q_{d a y}$, and the scaled volume change scaled by the absolute volume change (triangle), $q_{\text {vol }}$. The pdf for $q_{d a y}$ removes the second peak and shifts the distribution to the right. The pdf of the volume change $q_{v o l}$ has no second peak and the distribution is a bit symmetric. The tail parts of the pdfs show the fat tails. Inset of the Fig. 3 represents the log-log plot of the positive fat tails for the cumulative pdf. The tails of the cumulative pdf show the power law, $P_{>}(q) \sim q^{-\beta}$ with the exponent $\beta^{+}=4.05(7)$ for the positive tail and $\beta^{-}=4.37(7)$ for the negative tail regardless of the elimination of the intraday patterns.

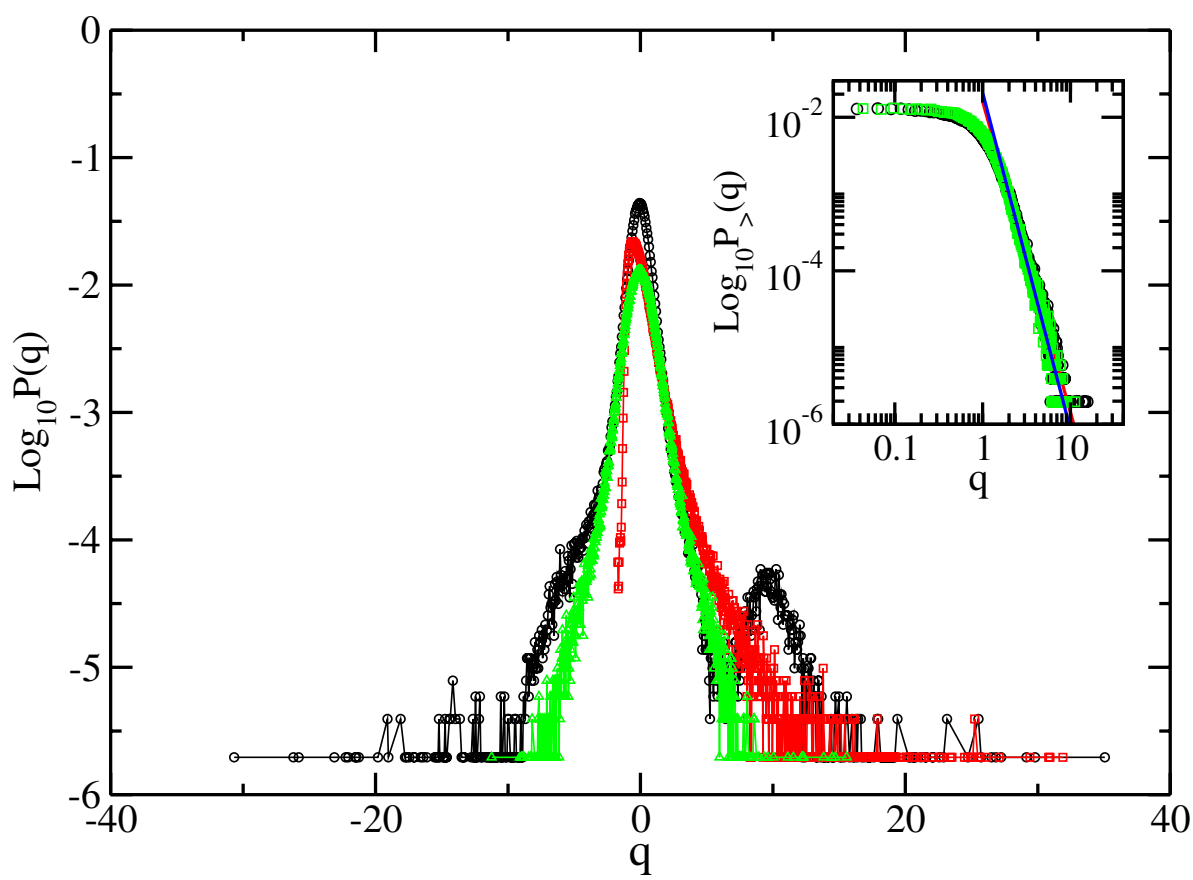

Fig. 3. The log-linear plot of the probability density function for the volume change of the original trading volume (circle), $q_{\text {org }}$, the volume change scaled by the intraday pattern of the trading volume itself (square), $q_{\text {day }}$, and the scaled volume change by the absolute volume change (triangle), $q_{v o l}$. The pdf for the volume change, $q_{\text {org }}$, shows a second peak around $q=10$. The pdf for $q_{d a y}$ removes the second peak and shifts the distribution to the right. The pdf of the volume change, $q_{v o l}$, has no second peak and the distribution function is symmetric. The tail parts of the pdfs show the fat tails. Inset represents the log-log plot of the positive fat tails for the cumulative pdf. The tails show the power law. The power exponent of the pdf for $q_{v o l}$ is larger than those for $q_{\text {org }}$ and $q_{\text {day }}$. 

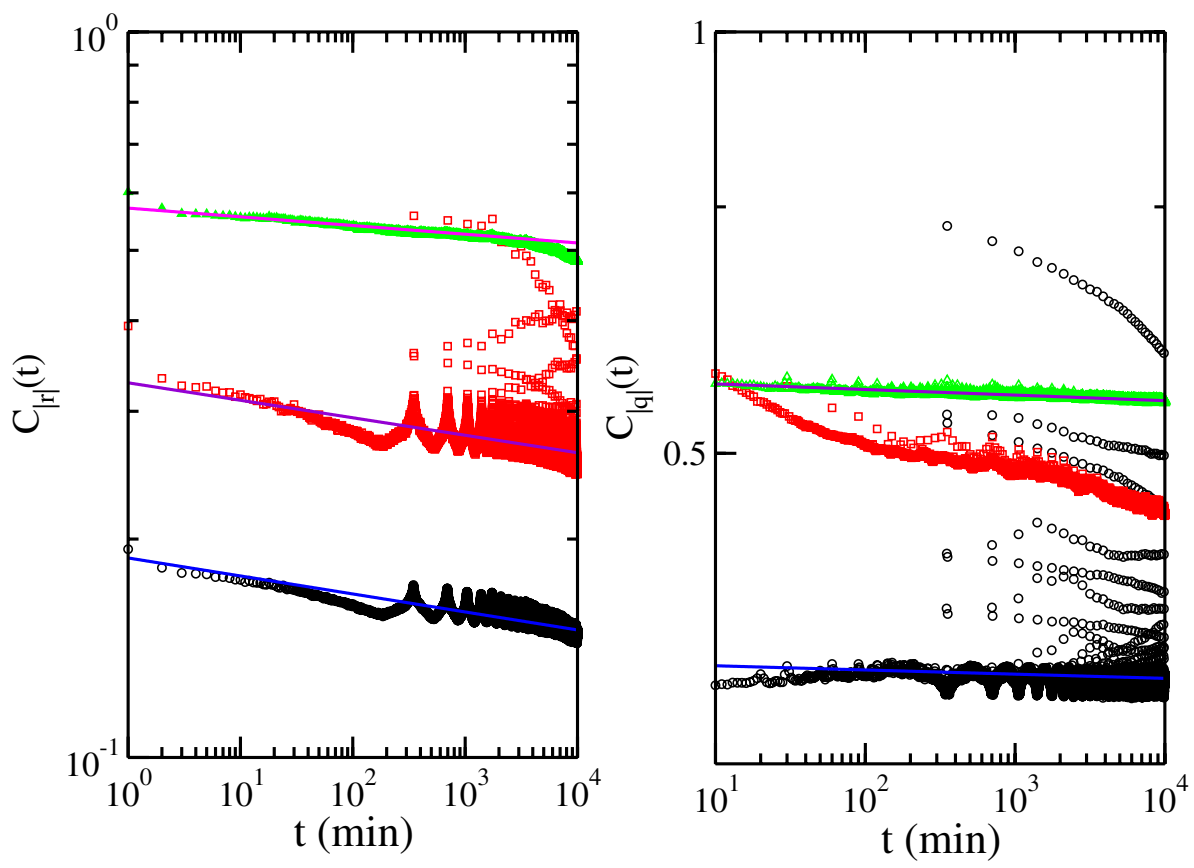

Fig. 4. Log-log plot of the autocorrelation function (a) for absolute return of the index: the absolute return of the original index, $r_{\text {org }}$ (circle); the absolute return scaled by the intraday pattern of index itself, $r_{\text {day }}$ (square); the absolute return scaled by the intraday pattern of absolute return, $r_{v o l}$ (triangle), and (b) for the absolute volume change; the volume change, $q_{\text {org }}$, (circle); the volume change, $q_{d a y}$, scaled by the intraday pattern of the volume change itself (square); the volume change, $q_{v o l}$, scaled by the absolute volume change(triangle). The solid lines are the least-square fits.

In Fig. 4 we present the log-log plot of the autocorrelation function for the absolute return obtained by the original return, $r_{\text {org }}$, and the scaled returns, $r_{d a y}$, $r_{v o l}$; and the autocorrelation function for the absolute volume change obtained by the original volume, $q_{\text {org }}$, and the scaled volume $q_{d a y}$, and $q_{v o l}$. We observed that the autocorrelation function of the absolute return showed long-time correlation and followed the power law, $C_{|r|}(t) \sim t^{-\gamma}$ for the return and $C_{|q|}(t) \sim t^{-\delta}$ for three types of the volume change. We obtained the power exponents as $\gamma($ org $)=$ $0.025(2), \gamma($ day $)=0.024(5), \gamma(\mathrm{vol})=0.025(1)$ for the return and $\delta($ org $)=0.003(5)$, $\delta($ day $)=0.027(7), \delta(\mathrm{vol})=0.004(2)$ for the volume change. The autocorrelation of the absolute return for the original return and the absolute return scaled by intraday patterns of the absolute return showed the periodic oscillation with one day period. However, in the autocorrelation of the absolute return for $r_{v o l}$, the periodic oscillation of the autocorrelation function disappeared. Therefore, we concluded the periodicity of the autocorrelation with a day period comes from the daily pattern of the return. Similar behaviors were observed for the autocorrelation of the absolute volume change in Fig. 4 (b). In the autocorrelation function of the absolute volume 
change, there were weak long-time correlations. We observed that the slopes of the $\log -\log$ plot for the autocorrelation function of the absolute volume change were very small. The long-time autocorrelations exist in the absolute return than in the absolute volume change.

\section{Conclusions}

We reviewed the stylized properties of the stock index. The probability distribution function of the return and the volatility deviate from the normal distribution and follow the power law at the fat tail. The return has the short-time memory, but the volatility shows the long-time memory in the autocorrelation function. The volume change also shows the fat tail in the probability distribution function. We also consider the effects of the intraday patterns in the analysis of the time series of the stock market. We consider two eliminating methods of the intraday patterns. We observed the probability distribution function, and the autocorrelation functions of the return and absolute return for the original time series and the time series eliminating the intraday patterns. The fat tails of the probability distribution of the return and volatility decay much faster when we eliminate the intraday patterns by the absolute return and absolute volume change. We observed that the daily oscillation of the autocorrelation was eliminated when we eliminate the intraday patterns by the absolute return and absolute volume change.

\section{Acknowledgments}

This work has been supported by the Research Fund of Inha University.

\section{References}

1. R. N. Mantegna and H. E. Stanley, Nature 376, 46 (1995).

2. R. N. Mantegna and H. E. Stanley, An Introduction to Econophysics: Correlations and Complexity in Finance (Cambridge University Press, New York, 2000), p. 20.

3. J. Voit, The Statistical Mechanics of Financial Markets (Springer, Berlin, 2001), p. 59.

4. S. Sinha, A. Chatterjee, A. Chakraborti, and B. K. Chakrabarti, Econophysics (WileyVCH, Morlenbach, 2011).

5. X. Gabaix, P. Gospikrishnan, V. Plerou, and H. E. Stanley, Nature 423, 26 (2003).

6. R. L. Axtell, Science 293, 1818 (2001).

7. R. Cont, Quant. Fin. 1, 223 (2001).

8. Z. Eisler and J. Kertesz, Physica A 343, 603 (2004).

9. V. Plerou and H. E. Stanley, Phys. Rev. E 76, 046109 (2007).

10. B. H. Hong, K. E. Lee, and J. W. Lee, Phys. Lett. A 361, 6 (2007).

11. J. W. Lee, K. E. Lee, and P. A. Rikvold, Physica A 364, 355 (2006).

12. B. H. Hong, K. E. Lee, J. K. Hwang, and J. W. Lee, Physica A 388, 863 (2009).

13. W. Li, F. Wang, S. Havlin, and H. E. Stanley, Phys. Rev. E 84, 046112 (2011).

14. K. E. Lee and J. W. Lee, J. Korean Phys. Soc. 47, 185 (2005).

15. J. W. Lee, J. Korean Phys. Soc. 56, 940 (2010).

16. K. E. Lee and J. W. Lee, J. Korean Phys. Soc. 50, 178 (2007). 
17. K. E. Lee and J. W. Lee, J. Korean Phys. Soc. 44, 668 (2004).

18. J. W. Lee, K. E. Lee, and. P. A. Rikvold, J. Korean Phys. Soc. 48, 123 (2006).

19. B. Podobnik, D. Horvatic, A. M. Petersen, and H. E. Stanley, PNAS 106, 22079 (2009)

20. A. Admati and P. Pfleiderer, Rev. Financial Studies 1, 3 (1988).

21. Y. Liu, P. Gopikrishnan, P. Cizeau, M. Meyer, C.-K. Peng, and H. E. Stanley, Phys. Rev. E 66, 1390 (1999).

22. F. Ren and W.-X. Zhou, Phys. Rev. E 81, 066107 (2010).

23. F. Wang, K. Yamasaki, S. Havlin, and H. E. Stanley, Phys. Rev. E 73, 026117 (2006).

24. F. Secuk and R. Gencay, Physica A 367, 375 (2006).

25. M. M. Cornett, T. V. Schwarz, and A. C. Szakmary, J. Bank. Finan. 19, 843 (1995).

26. R. A. Wood, T. H. McIntosh, J. K. Ord, J. Finance 40, 723 (1985).

27. P. Gopikrishnan, V. Plerou, X. Gabaix, and H. E. Stanley, Phys. Rev. E 62, R4493 (2000).

28. T. Kaizoji and M. Nuki, Fractal 12, 49 (2004). 